\title{
Doorway Flows Induced by the Combined Effects of Natural and Forced Ventilation in a Three Compartment Assembly
}

\author{
H. PRETREL, and L. AUDOUIN \\ Institut de Radioprotection et de Sûreté Nucléaire (IRSN) \\ Laboratoire d'Expérimentation des Feux (DPAM/SERCI/LEF) \\ Centre de Cadarache, 13115 Cedex St Paul-lez-Durance, France
}

\begin{abstract}
This research deals with the combined effects of fire and mechanical ventilation on the bidirectional flow occurring at a doorway for a fire scenario with a three compartment assembly. Based on large-scale fire tests, an experimental campaign has been carried out in order to investigate the effect of the ventilation rate and the fire heat release rate on doorway flow. The analysis focused on the velocity profiles at the doorway, the location of the neutral plane and the amplitude of the velocities entering and leaving the fire compartment. The conclusions point out the significant effects of the ventilation on the features of the doorway flow. The mechanical ventilation modified the relative contribution of inflows and outflows and might stop the release of smoke. Moreover, the position of the doorway in relation to the fire location and the direction of the ventilation flow have to be considered. A correlative approach has been proposed to support the analysis.
\end{abstract}

KEYWORDS: compartment fires, smoke, doorway, mechanical ventilation, buoyancy, pressure.

\section{NOMENCLATURE LISTING}

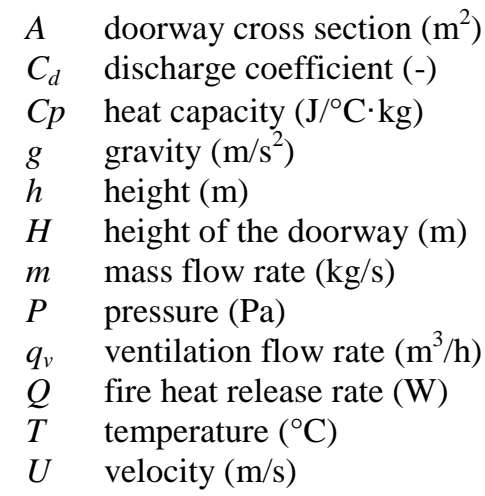

$\begin{array}{ll}z & \text { vertical position }(\mathrm{m}) \\ \text { Greek } & \\ \rho & \text { gas density }\left(\mathrm{kg} / \mathrm{m}^{3}\right) \\ \text { superscripts and subscripts } \\ N & \text { neutral plane } \\ s & \text { static pressure } \\ O & \text { without ventilation } \\ * & \text { dimensionless variable } \\ H & \text { doorway height } \\ i & \text { inflow } \\ o & \text { outflow } \\ \infty & \text { ambient conditions }\end{array}$

\section{INTRODUCTION}

For fire safety assessment in nuclear installations, smoke movement is a key phenomenon. Fire smoke may impair major safety equipment as it is a propagation vector of heat and soot. Typical unintended events are the clogging of high efficiency filters (HEPA) located in the ventilation network or the failure of electrical or electronic devices. In addition, fire scenarios in nuclear installations involve mostly ventilated compartments. So, smoke movement may have a feedback effect on the combustion regime; either by the process of smoke filling in the compartment, or by modifying the 'fresh' air entrained into the fire room.

One aspect of smoke movement that is frequently investigated by the scientific community is the doorway flow (vertical vent flow). Because this is a region of transfer between rooms, an appropriate description of such flow is needed in order to accurately assess the amount of smoke released toward the installation and the amount of 'fresh' air brought to the fire and governing the regime of combustion. For open atmosphere scenario, the physical mechanism governing the flow is the buoyancy due to temperature difference between the two sides of the doorway. For mechanically ventilated compartments, the driving force associated with the ventilation (i.e., the difference of static pressure between rooms) is added to buoyancy.

In open atmosphere (without the effect of mechanical ventilation), scientific contributions on doorway flows have been numerous. A theoretical basis for the flow description has been proposed since the 
seventies [1-5]. Supported by experiments, correlations have been proposed to estimate the mass flow rate through the opening [6-8]. Research is still underway focusing on the generalization of correlations $[9,10]$, comparisons with numerical codes $[11,12]$ and experimental investigation using more detailed measurement techniques such as PIV (particle image velocimetry) [13].

The coupling between natural and forced ventilation has been much less investigated. The main applications for which research has been conducted are the use of positive pressure ventilation (PPV) techniques for smoke control [1], the interest of forced ventilated fire scenarios [14,15] or the HVAC (heat ventilation air conditioning) system in buildings [2].

This paper focuses on the coupled effects of fire and mechanical ventilation. Based on large-scale experiments, it brings a new set of experimental data showing a comprehensive description of the flow. In addition, the specific fire scenario considered (three successive rooms connected with doorways) allows an investigation of the effect of the position of the fire room relative to the ventilation flow through the doorways.

\section{THEORETICAL BACKGROUND}

The flow at a doorway results from the contributions of two terms, the hydrostatic pressure difference resulting from buoyancy (difference of density $\Delta \rho(z)$ ), which varies with elevation (z), and the static pressure induced by the ventilation network, which is constant over height:

$$
\Delta P(z)=g \Delta \rho(z)\left(z-h_{N}^{o}\right)+\Delta P_{S}
$$

Without additional static pressure, a bidirectional flow occurs at the doorway and the outflow and inflow rates are balanced (Fig. 1). The height $h_{N}{ }^{o}$, referred to as the neutral plane, is the location of the interface between the two flows where the static pressure is equal to zero (only due to natural ventilation).

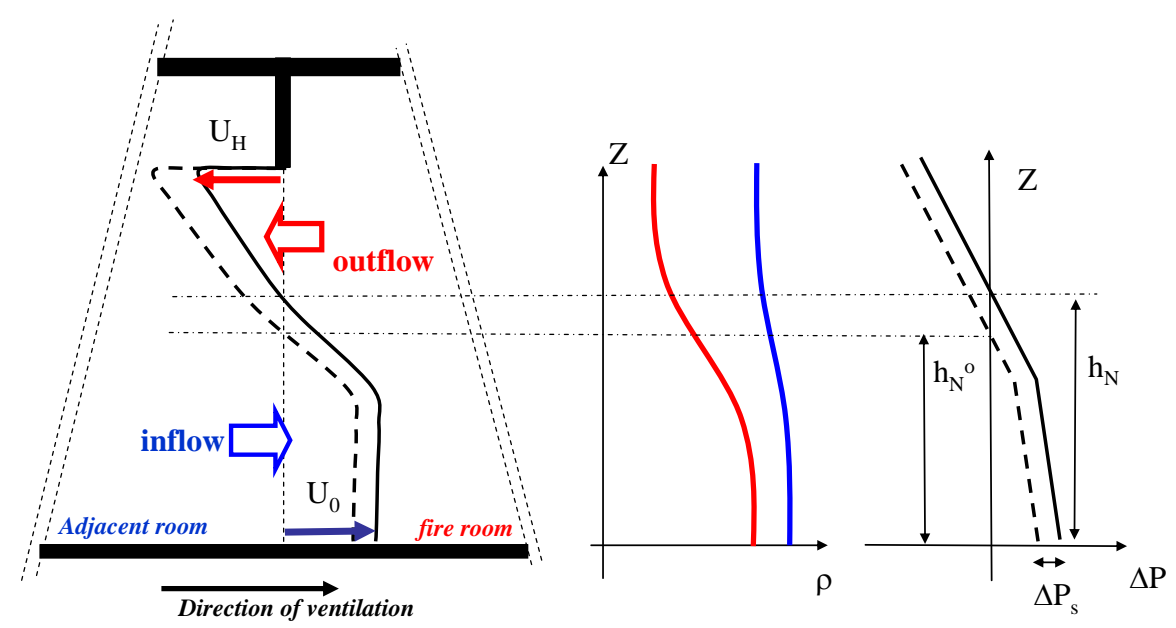

Fig. 1. Schematic representation of doorway flow and main parameters,

When, the effect of the forced ventilation is added, the effective neutral plane, $h_{N}$, is the height where the pressure difference is zero with both pressure contributions (natural and forced ventilation). It can be expressed as:

$$
\Delta P_{S}+g \Delta \rho\left(h_{N}-h_{N}^{o}\right)=0 \quad \text { or } \quad h_{N}^{*}=h_{N}^{o, *}-\frac{\rho}{\Delta \rho} \frac{\rho_{\infty}}{\rho} \Delta P_{S}^{*}
$$

with $\Delta P_{S}^{*}=\frac{\Delta P_{S}}{\rho_{\infty} g H}, \quad h_{N}^{o, *}=\frac{h_{N}^{o}}{H} \quad$ and $\quad h_{N}^{*}=\frac{h_{N}}{H}$ 
The first term in Eq. 1 depends on the fire only and the second term on the coupling between fire and mechanical ventilation. Based on Bernoulli's relation, the local horizontal velocity is:

$U(z)=\sqrt{\frac{2|\Delta P(z)|}{\rho(z)}} \quad$ and $\quad U_{Z}^{*}=\frac{U(z)}{\sqrt{g H}}=\sqrt{2\left|\frac{\Delta \rho}{\rho} \frac{\left(z-h_{N}^{o}\right)}{H}+\frac{\rho_{\infty}}{\rho} \Delta P_{S}^{*}\right|}$

Expressions for the maximum and minimum velocities near the top and bottom of the doorway are given as:

$\frac{1}{2} U_{H}^{* 2}=\left|\frac{\Delta \rho}{\rho}\left(1-h_{N}^{o^{*}}\right)+\frac{\rho_{\infty}}{\rho} \Delta P_{S}^{*}\right| \quad$ and $\quad \frac{1}{2} U_{0}^{* 2}=\left|\frac{\Delta \rho}{\rho} \frac{\rho}{\rho_{\infty}} h_{N}^{o, *}-\Delta P_{S}^{*}\right|$

The total mass flow rate is then formulated as:

$\dot{m}_{i}^{*}=C_{d} \int_{0}^{h_{N}^{*}} U^{*} d z^{*} \quad$ and $\quad \dot{m}_{o}^{*}=C_{d} \int_{h_{N}}^{1} U * \frac{\rho}{\rho_{\infty}} d z^{*}$

The purpose of the present work is to investigate through experimentation how the presence of fire and ventilation affects the variables: $h_{N}{ }^{*}, U_{0}{ }^{*}, U_{H}{ }^{*}, m_{i}{ }^{*}$ and $m_{o}{ }^{*}$.

\section{FIRE EXPERIMENTS}

\section{Facility Description}

The international PRISME project dealing with fire smoke propagation in nuclear installations provided an opportunity to perform gas fire tests in the DIVA, a large scale facility of IRSN [14]. The scenario presented in Fig. 2 involves a set of three compartments (named $L_{1}, L_{2}, L_{3}$ ) entirely closed and connected to each other by two doorways (named $D_{12}$ and $D_{23}$ ). The three rooms are identical ( $4 \mathrm{~m}$ in height $\times 5 \mathrm{~m} \times$ $6 \mathrm{~m}$ ). The fire room $L_{2}$ and the adjacent room $L_{3}$ are thermally insulated with rock-wool panels. The fire source is a gas propane burner located in the center of the room.

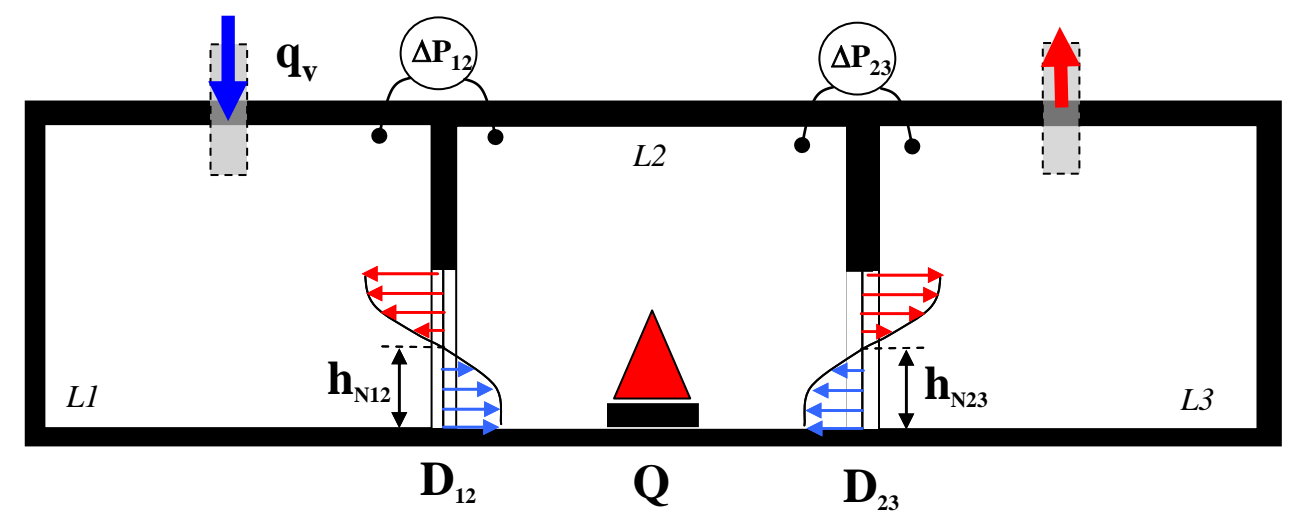

Fig. 2. Configuration of the fire experiments.

Doorways are located at the center of each separating wall. The lintel is at $1.86 \mathrm{~m}$ and there is no sill. The dimensions, identical for both doorways, are $2,140 \mathrm{~mm}$ in height and $810 \mathrm{~mm}$ in width.

The ventilation network comprises an admission duct on the ceiling of room $L_{l}$ and an exhaust duct on the ceiling of room $L_{3}$. The direction of the ventilation air flows goes from room $L_{1}$ to room $L_{3}$. Considering the flow direction, doorway $D_{12}$ is the upstream one and doorway $D_{23}$, the downstream one. This specific 
configuration makes it possible to investigate the flow through the doorway, depending on its position (upstream or downstream) relative to the forced flow through the fire room.

The fire is produced by a gas burner characterized by the propane flow rate measured with a mass flowmeter. The ventilation flow rate is measured with pitot probes in both ventilation ducts. The static pressure between rooms is measured with membrane pressure transducers.

\section{Experimental Determination of the Doorway Flow}

Each doorway is equipped with seven bidirectional velocity probes and seven Type- $K$ thermocouples positioned at the centerline of the doorways. The bidirectional probes have been tested in a wind tunnel in order to determine the probe coefficient. The positions of the probes are indicated in Fig. 3. The total mass flow rate is obtained from the integration of the velocity profiles taking into account the change of temperature.

$$
\dot{m}=C w\left(\rho\left(T_{0}\right) z_{0} u_{o}+\sum_{i=0}^{5} \rho\left(\frac{T_{i+1}+T_{i}}{2}\right)\left(z_{i+1}-z_{i}\right)\left(\frac{u_{i+1}+u_{i}}{2}\right)+\rho\left(T_{6}\right)\left(H-z_{6}\right) u_{6}\right)
$$

The sill and soffit terms account for more accuracy near the boundaries. $W$ is the door width. The $C$ coefficient takes into account the fact that the velocity profiles are not flat over the whole doorway crosssection while the probes are located in the centerline of the door. It has been determined experimentally at ambient temperature for several air flow rates and in the two directions. The average measured coefficient for both doorways is $0.87 \pm 0.08$.
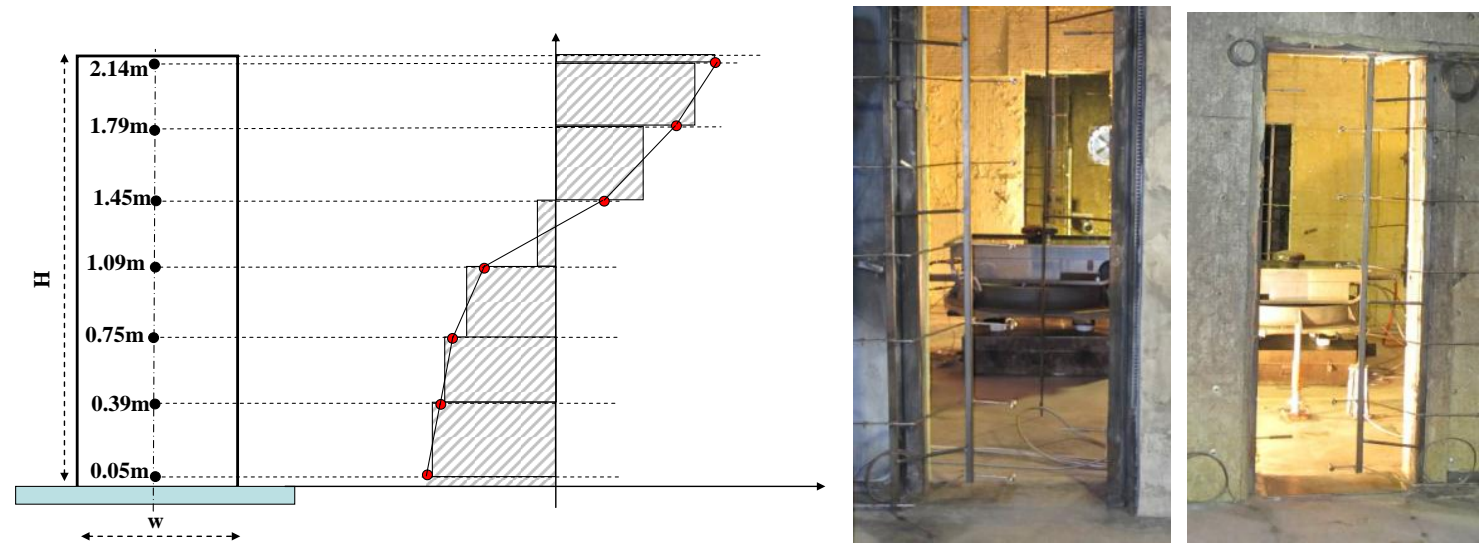

Fig. 3. Dimensions and pictures of the doorways.

\section{Fire Test Matrix}

Seven fire tests are considered. The fire heat release rate (HRR) varies from 100 to $400 \mathrm{~kW}$ and the ventilation flow rate varies from 500 to $3,100 \mathrm{~m}^{3} / \mathrm{h}$ (Fig. 4). The fire HRR is obtained from the propane volumetric flow rate measurement multiplied by the gas density $\left(\rho_{C 3 H 8}=1.8 \mathrm{~kg} / \mathrm{m}^{3}\right)$ and the effective combustion enthalpy for propane $\left(\Delta H_{c}=46 \mathrm{MJ} / \mathrm{kg}\right)$. Dimensionless variables for both ventilation flow rate and heat release rate are defined below:

$q_{v}^{*}=\frac{q_{v}}{\sqrt{g H} A}$ and $Q^{*}=\frac{Q}{\rho_{\infty} T_{\infty} C_{P} \sqrt{g H} A}$

The ventilation rate $q_{v}$, used to characterize the test, is the steady state magnitude measured before ignition. During the combustion phase, this rate can vary. 


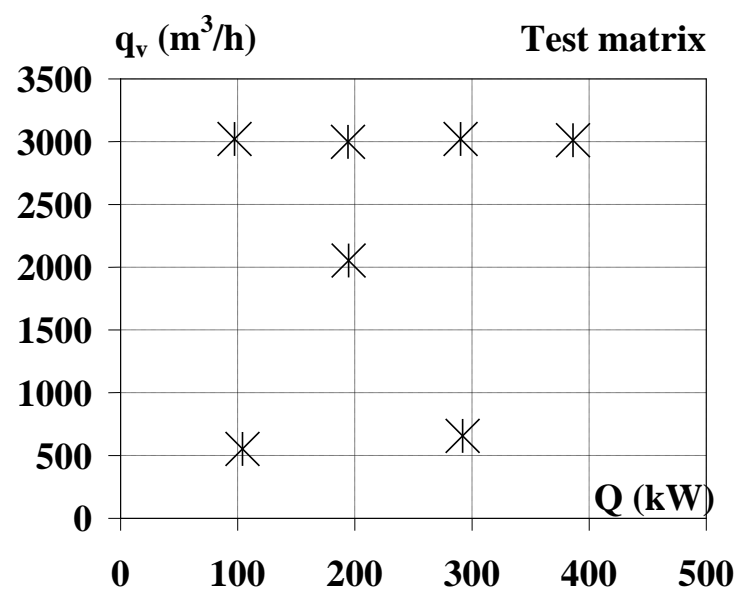

Fig. 4. Test matrix graph.

\section{Experimental Procedure}

First the ventilation network is set-up in order to achieve the targeted ventilation flow rate. Then the gas burner is turned on so that it generates a natural convection flow at the doorways. Figure 5 illustrates the time variation of ventilation rates, the fire HRR and the pressure within the rooms. The fire HRR is constant over the test duration. On the other hand, the ventilation flow rate, set to a constant value before ignition, does vary during the combustion phase, due to the change of gas temperature within the rooms.

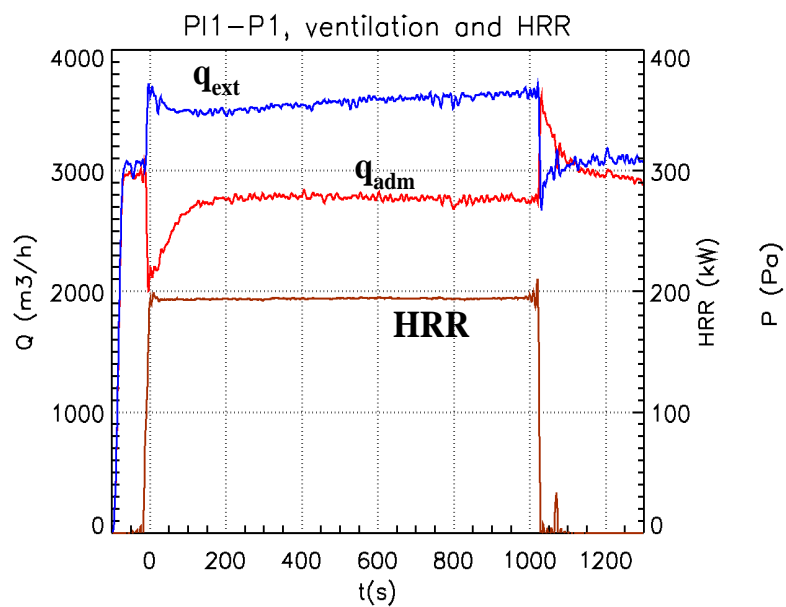

(a)

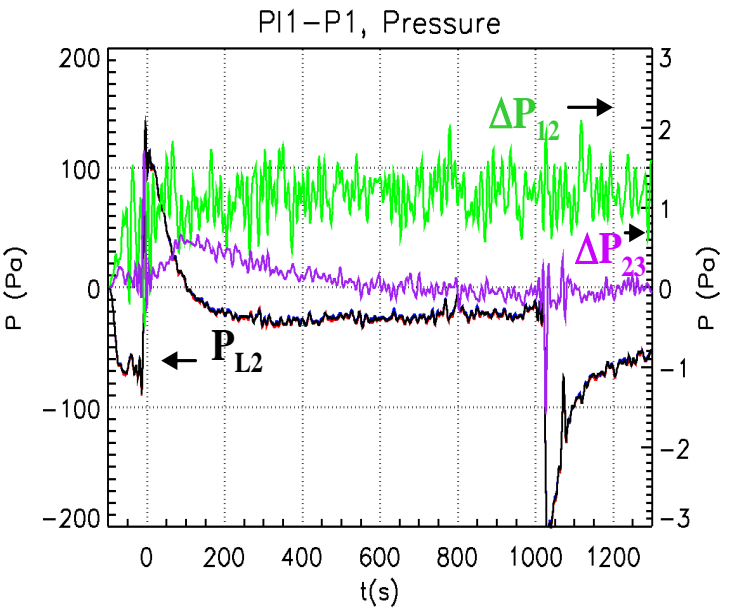

(b)

Fig. 5. Time variation of the ventilation flow rates (admission and exhaust) and: (a) HRR; (b) pressure within the rooms.

The flows at both doorways show the typical expected bidirectional flow behavior (Fig. 6). The test is stopped (gas burner turned off) once the flow reaches a steady state regime in term of the velocity at the doorways and the gas temperature at the doorways and within the rooms. This is achieved after about 15 min of combustion for all tests. 

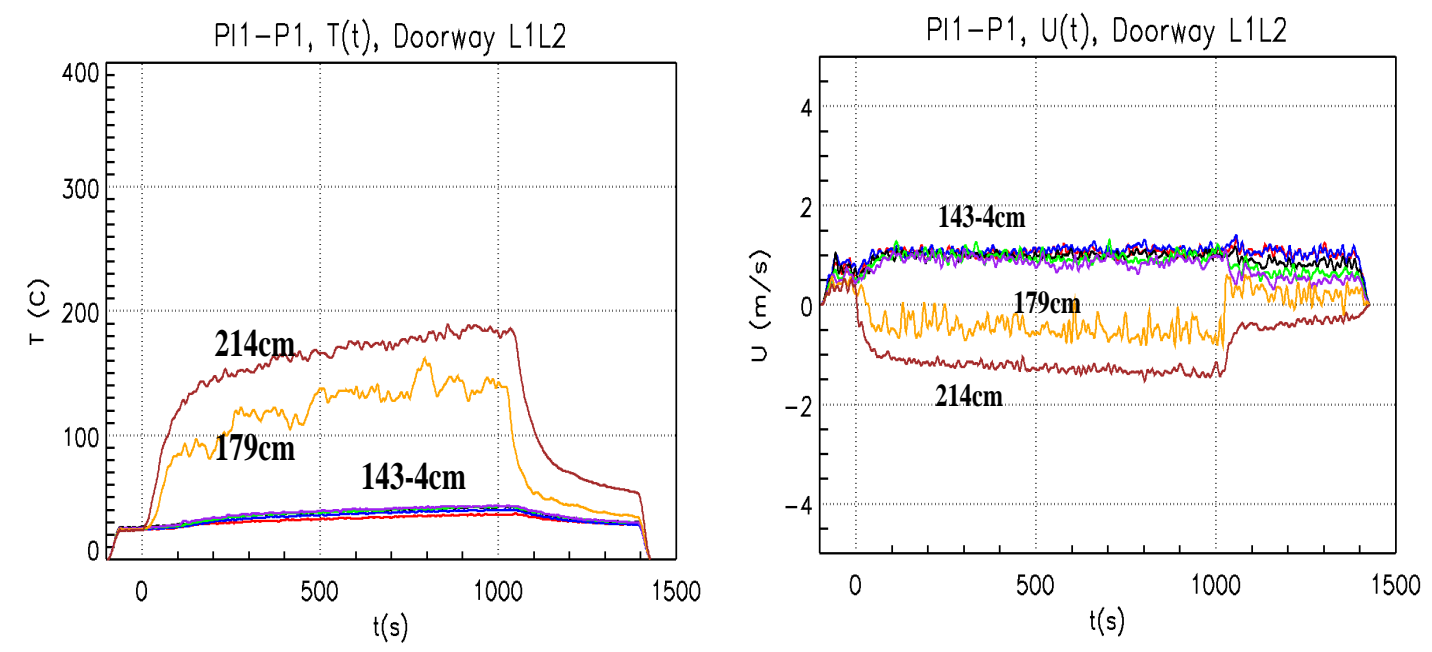

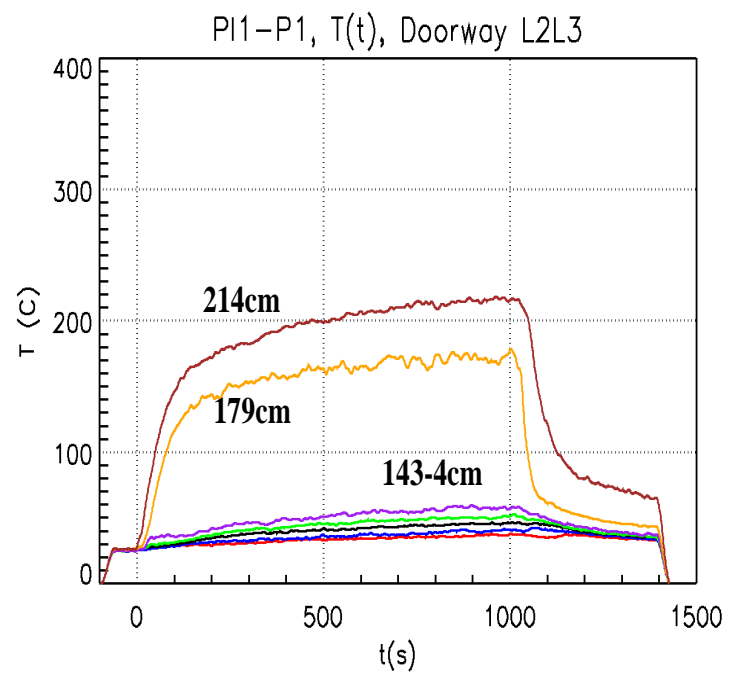

(a)

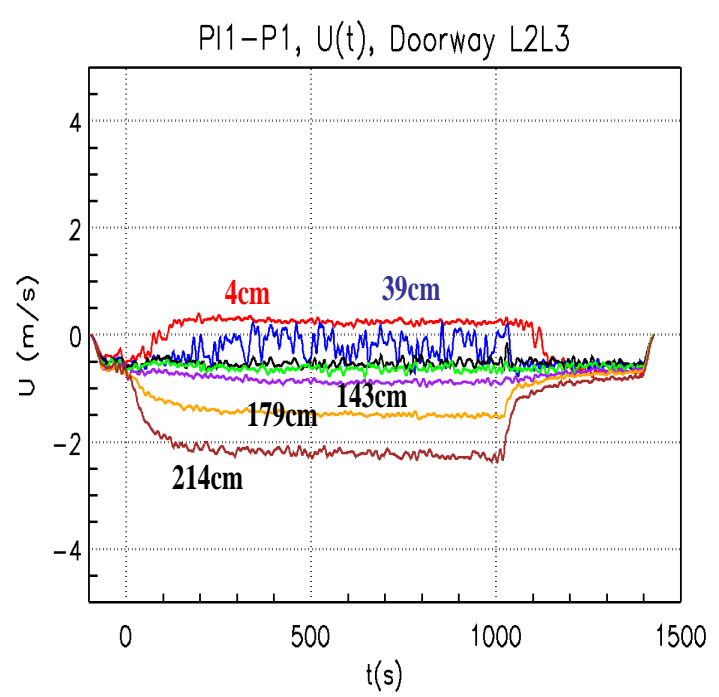

(b)

Fig. 6. Time variation of: (a) gas temperatures; (b) gas velocities at the doorways $\mathrm{D}_{12}$ and $\mathrm{D}_{23}$.

\section{DATA ANALYSIS}

\section{Static Pressure between Rooms}

The mechanical ventilation leads to successive pressure drops between rooms considered as nodes along the ventilation network. Before ignition, the pressure cascade through the ventilation network shows a typical decrease: the pressure difference is proportional to the ventilation flow rate. This behavior is schematically illustrated in Fig. 7. The order of magnitude of the pressure difference is about $1 \mathrm{~Pa}$. During the combustion phase, the fire behaves as a pressure generator. Depending on the fire HRR, it may reverse the static pressure difference with the upstream room $L_{l}$ and significantly increase the downstream flow rate. This phenomenon is an important issue for the understanding of the smoke flow between compartments. The fire has two effects in the smoke flow: firstly, it raises the gas temperature, which then brings on buoyancy forces; secondly it induces static pressure variations because of the confinement of the fire room. This second effect is closely linked to the tightness and dimension of the rooms compared to the fire HRR. This is typical to scenarios encountered in the nuclear industry [16]. 


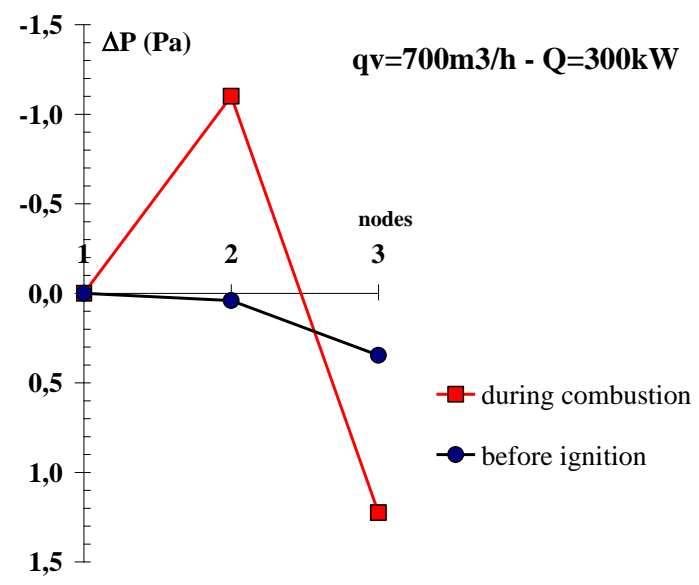

Fig. 7. Pressure cascade before ignition and during the combustion phase.

\section{Velocity Profiles}

Through the seven experiments, the effect of the ventilation rate and the fire HRR on the velocity profiles is analyzed during the steady state regime.

Fig. 8 shows the change of the velocity profiles due to the fire HRR for tests performed with ventilation flow rate of $3,100 \mathrm{~m}^{3} / \mathrm{h}$. The rise of the fire HRR increases the value of the outflow of smoke at both doorways and consequently lowers the location of the neutral plane. The effect of the fire HRR on the inflow of 'fresh' air is less pronounced or even negligible. In addition, Fig. 8 points out the asymmetry between the two doorway flows. At the upstream (relative to the fire room) doorway $\mathrm{D}_{12}$, the flow shows a typical profile with a flat shape for the inflow and a linear increase shape for the outflow. This is the typical behavior expected for the natural ventilation case. At the downstream doorway $\mathrm{D}_{23}$, the flow shows a different shape with continuous increase of the velocity from bottom to top.
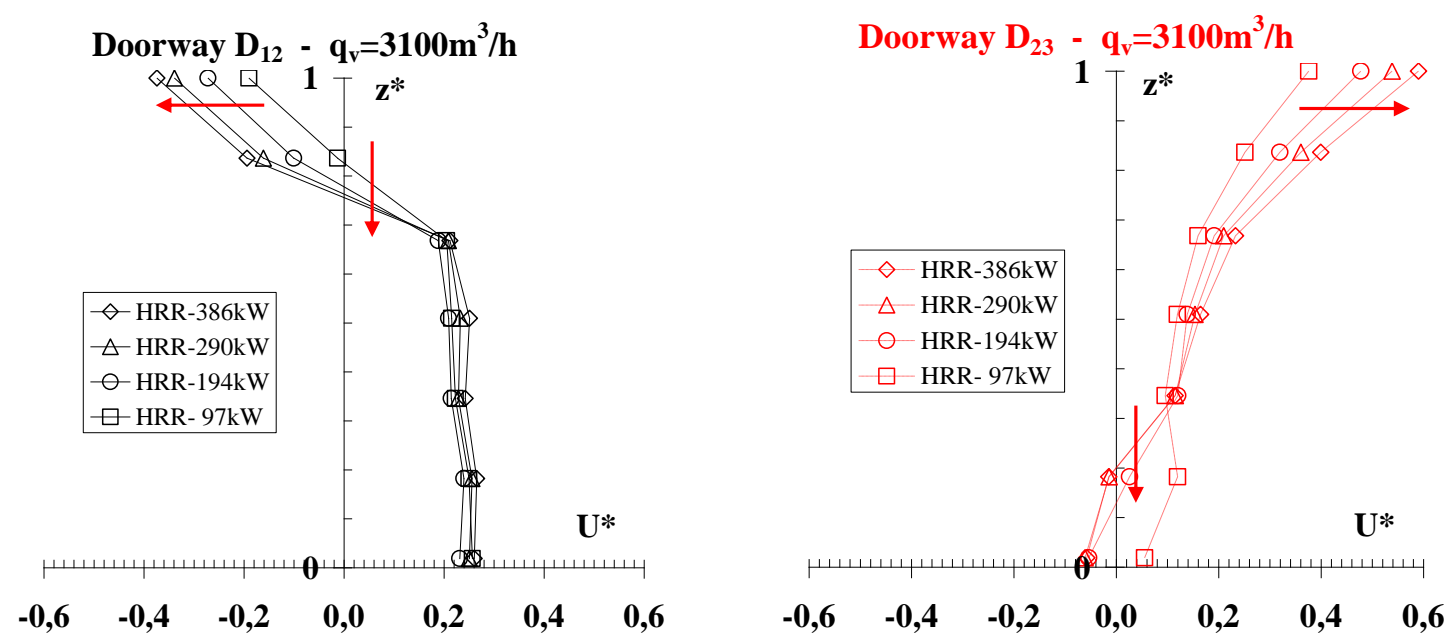

Fig. 8. Effect of the fire HRR on the velocity profiles at doorways for a given ventilation flow rate $\left(3,100 \mathrm{~m}^{3} / \mathrm{h}\right)$.

Fig. 9 and Fig. 10 point out the effect of the ventilation flow rate on the doorway flow for two fire HRRs $(100 \mathrm{~kW}$ and $290 \mathrm{~kW})$. The main effect of the forced ventilation is to shift the value of the inflow. At both doorways and for both fire HRRs, this influence is significant. At the upstream doorway $\left(\mathrm{D}_{12}\right)$, the rise of the ventilation flow rate increases the inflow velocity and consequently moves up the neutral plane. 
Inversely, at the downstream doorway $\left(\mathrm{D}_{23}\right)$, the flow rate increase contributes to reduce the inflow and moves the neutral plane down. For instance, for the test $\left(100 \mathrm{~kW}\right.$ and $\left.3,100 \mathrm{~m}^{3} / \mathrm{h}\right)$, the inflow of 'fresh' air from the downstream side completely disappears.
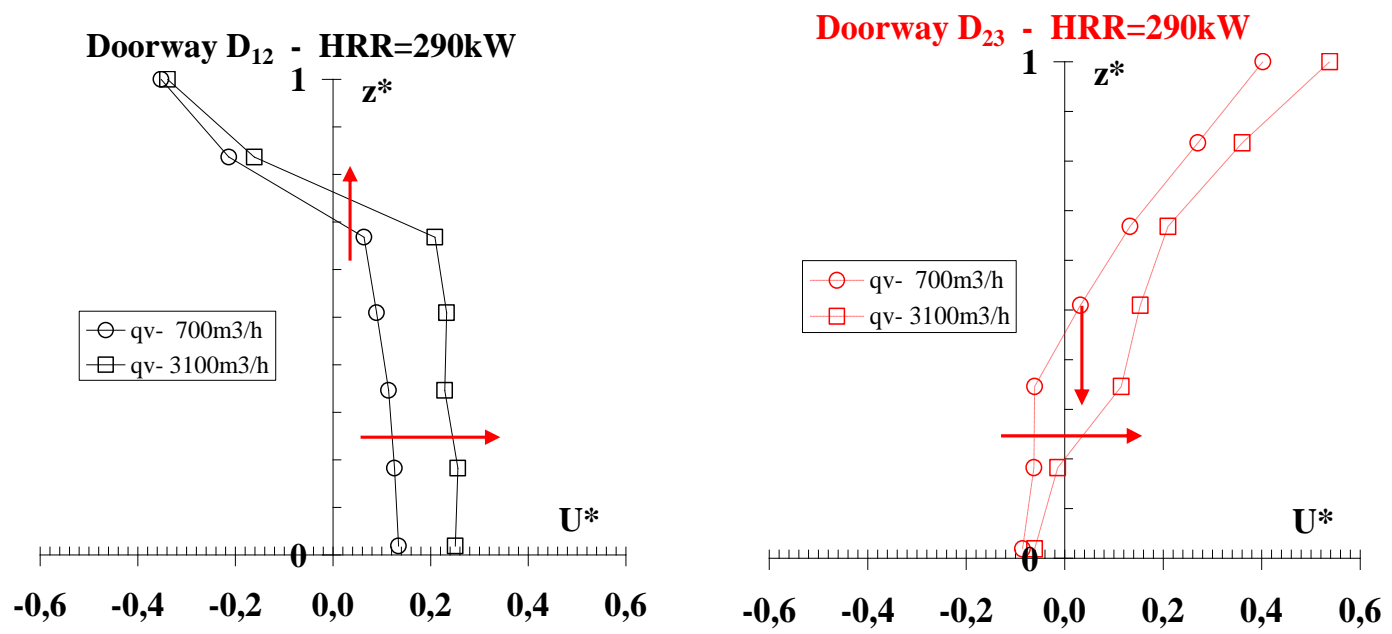

Fig. 9. Effect of the ventilation flow rate on the velocity profiles at doorways for a given fire HRR $(290 \mathrm{~kW})$.
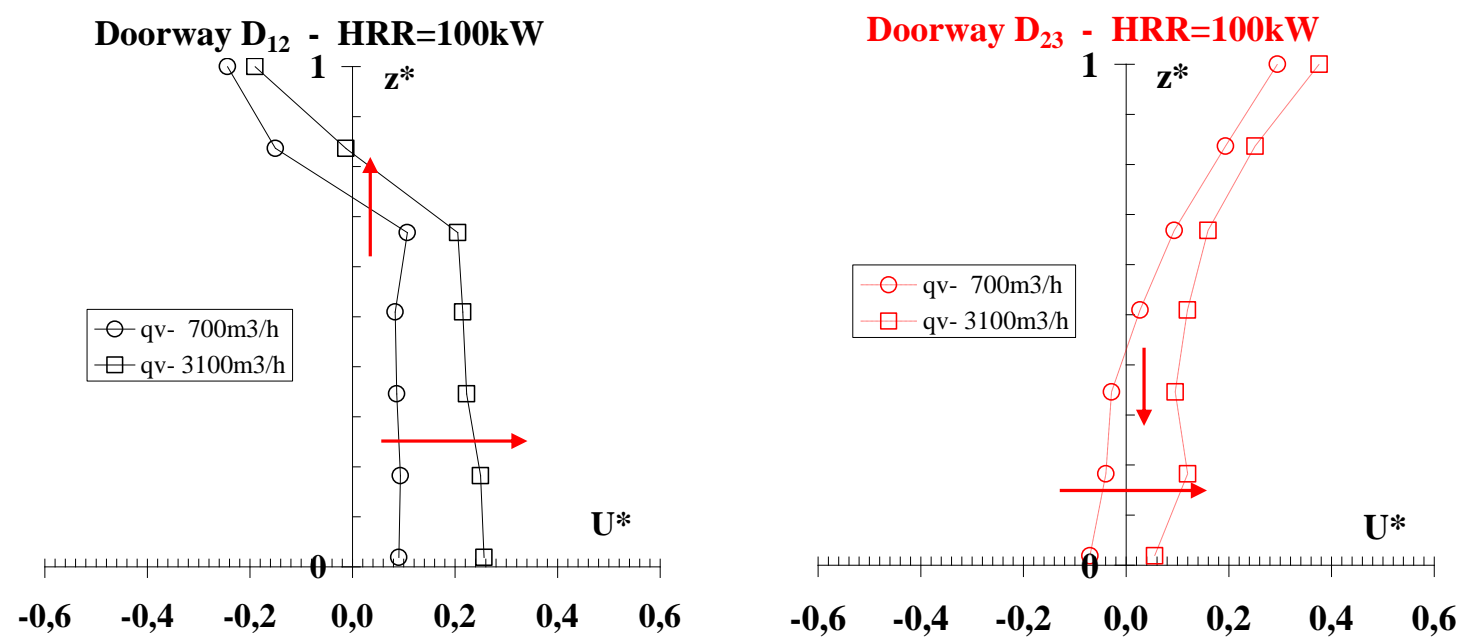

Fig. 10. Effect of the ventilation flow rate on the velocity profiles at doorways for a given fire HRR $(100 \mathrm{~kW})$.

\section{CORRELATIVE APPROACH}

\section{Methodology}

A correlative approach is proposed to generalize the effect of forced ventilation and fire on the three dimensionless variables: the neutral plane $h_{N}{ }^{*}$ and the top and bottom velocities $U_{H}{ }^{*}, U_{0}{ }^{*}$. According to the theoretical approach (Eq. 2 and Eq. 4), the three variables can be expressed as a function of the four parameters $h_{N}{ }^{*}, \Delta \rho / \rho, \rho_{\text {inf }} f \rho$ and $\Delta P_{s}^{*}$ such as: 


$$
\begin{aligned}
h_{N}^{*} & =h_{N}^{o_{*}^{*}}+\delta \frac{\rho}{\Delta \rho} \frac{\rho_{\infty}}{\rho} \Delta P_{S}^{*} \\
U_{H}^{*} & =\sqrt{2\left|\frac{\Delta \rho}{\rho}\left(1-h_{N}^{o *}\right)-\delta \frac{\rho_{\infty}}{\rho} \Delta P_{S}^{*}\right|} \quad \text { with } \delta=1 \text { for doorway } \mathrm{D}_{12} \text { and } \delta=-1 \text { for } \mathrm{D}_{23} \\
U_{0}^{*} & =\sqrt{2\left|\frac{\Delta \rho}{\rho} \frac{\rho}{\rho_{\infty}} h_{N}^{o *}+\delta \Delta P_{S}^{*}\right|}
\end{aligned}
$$

The objective of this approach is to propose empirical correlations for the four parameters $\left(h_{N}{ }^{*}, \Delta \rho / \rho\right.$, $\left.\rho_{\text {inf }} \rho, \Delta P_{s}^{*}\right)$ such that the values of $h_{N}{ }^{*}, U_{H}{ }^{*}$ and $U_{0} *$ determined by the relationships in Eq. 8, match the corresponding measured values.

The correlation for the parameter $h_{N}{ }^{0} *$ proposed below (Eq. 9) is such that it provides a good fit with the data and with general formulations available in the literature for the simple case [17]. Since the elevation of the neutral plane $h_{N}{ }^{\circ}$ is only due to the natural ventilation induced by the fire, the proposed correlation depends only on the fire HRR and does not depend on the ventilation rate or on the doorway relative position (upstream and downstream):

$h_{N}^{o, *}=D_{0}\left(1+D_{1} Q^{* D_{2}}\right)$ with $D_{0}=0,65 ; D_{1}=-0,5$ and $D_{2}=1$

The correlations for the other three parameters $\Delta \rho / \rho, \rho_{\text {inf }} f \rho$ and $\Delta P_{s}^{*}$, are expressed as a function of the variables $h_{N}{ }^{*}, U_{H}{ }^{*}$ and $U_{0}{ }^{*}$ on the basis of the previous system of equation (Eq. 8) such as:

$$
\frac{\Delta \rho}{\rho}=\frac{U_{H}^{* 2}}{2\left(1-h_{N}^{*}\right)} \quad ; \quad \frac{\rho_{\infty}}{\rho}=\frac{U_{H}^{* 2}}{U_{0}^{* 2}} \frac{h_{N}^{*}}{1-h_{N}^{*}} \quad ; \quad \Delta P_{S}^{*}=\frac{U_{0}^{* 2}}{2}\left(1-\frac{h_{N}^{O *}}{h_{N}^{* *}}\right)
$$

These parameters are computed from the measured values of $h_{N}^{*}, U_{H} *$ and $U_{0} *$ and the correlation (Eq. 9) for the neutral plane $h_{N}{ }^{0} *$. Their variations are presented in Fig. 11. The three parameters depend on the fire HRR, the ventilation rate and the position of the doorway (upstream $D_{12}$ or downstream $D_{23}$ ).

Finally, three correlations are proposed for the parameters $\Delta \rho / \rho, \rho_{\alpha} / \rho$ and $\Delta P_{s}^{*}$, as functions of the ventilation rate $q_{v}{ }^{*}$ and the fire heat release $Q^{*}$ :

$$
\Delta P_{S}^{*}=A_{0} q_{v}^{* A_{1}}\left[1+A_{2} Q^{* A_{3}}\left(1+A_{4} q_{v}^{* A_{5}}\right)\right] \quad \frac{\rho_{\infty}}{\rho}=1+B_{0} Q^{* B_{1}}\left(1+B_{2} q_{v}^{* B_{3}}\right) \quad \frac{\Delta \rho}{\rho}=C_{0} Q^{* C_{1}}\left(1+C_{2} q_{v}^{* C_{3}}\right)
$$

The forms of these correlations satisfy the physical boundary conditions (for $Q=0$ and $q_{v}=0 ; \Delta \rho / \rho=0$; $\rho_{\alpha} \rho=1, \Delta P_{s}{ }^{*}=A_{0} q_{v}{ }^{* 2}$ ). The empirical coefficients, given in Table 1 , are determined by fitting the correlations to the experimental data.

\begin{tabular}{|c|c|c|c|c|c|c|c|c|c|c|c|c|c|c|}
\hline & \multicolumn{6}{|c|}{$\Delta P_{s}^{*}$} & \multicolumn{4}{|c|}{$\rho_{\alpha} / \rho$} & \multicolumn{4}{|c|}{$\Delta \rho / \rho$} \\
\hline & $\mathrm{A}_{0}$ & $\mathrm{~A}_{1}$ & $\mathrm{~A}_{2}$ & $\mathrm{~A}_{3}$ & $\mathrm{~A}_{4}$ & $\mathrm{~A}_{5}$ & $\mathrm{~B}_{0}$ & $\mathrm{~B}_{1}$ & $\mathrm{~B}_{2}$ & $\mathrm{~B}_{3}$ & $\mathrm{C}_{0}$ & $\mathrm{C}_{1}$ & $\mathrm{C}_{2}$ & $\mathrm{C}_{3}$ \\
\hline$D_{12}$ & 1.5 & 2 & -1 & 0.2 & 0.1 & 1 & 35 & 0.4 & -2 & 0.5 & 0.96 & $\begin{array}{l}0.7 \\
\end{array}$ & 1 & 0.8 \\
\hline$D_{23}$ & -1.5 & 2 & -1 & 0.09 & 0.1 & 1 & 35 & 0.4 & 2 & 0.5 & 0.5 & 0.6 & 2.2 & 0.8 \\
\hline
\end{tabular}

These correlations are then introduced in the three relationships given as Eq. 8 and predictive values for $h_{N}^{*}, U_{H}{ }^{*}$ and $U_{0}{ }^{*}$ can be calculated. They must be compared to the experimental results to support the analysis.

Table 1. Coefficients for the three correlations of Eq. 11. 

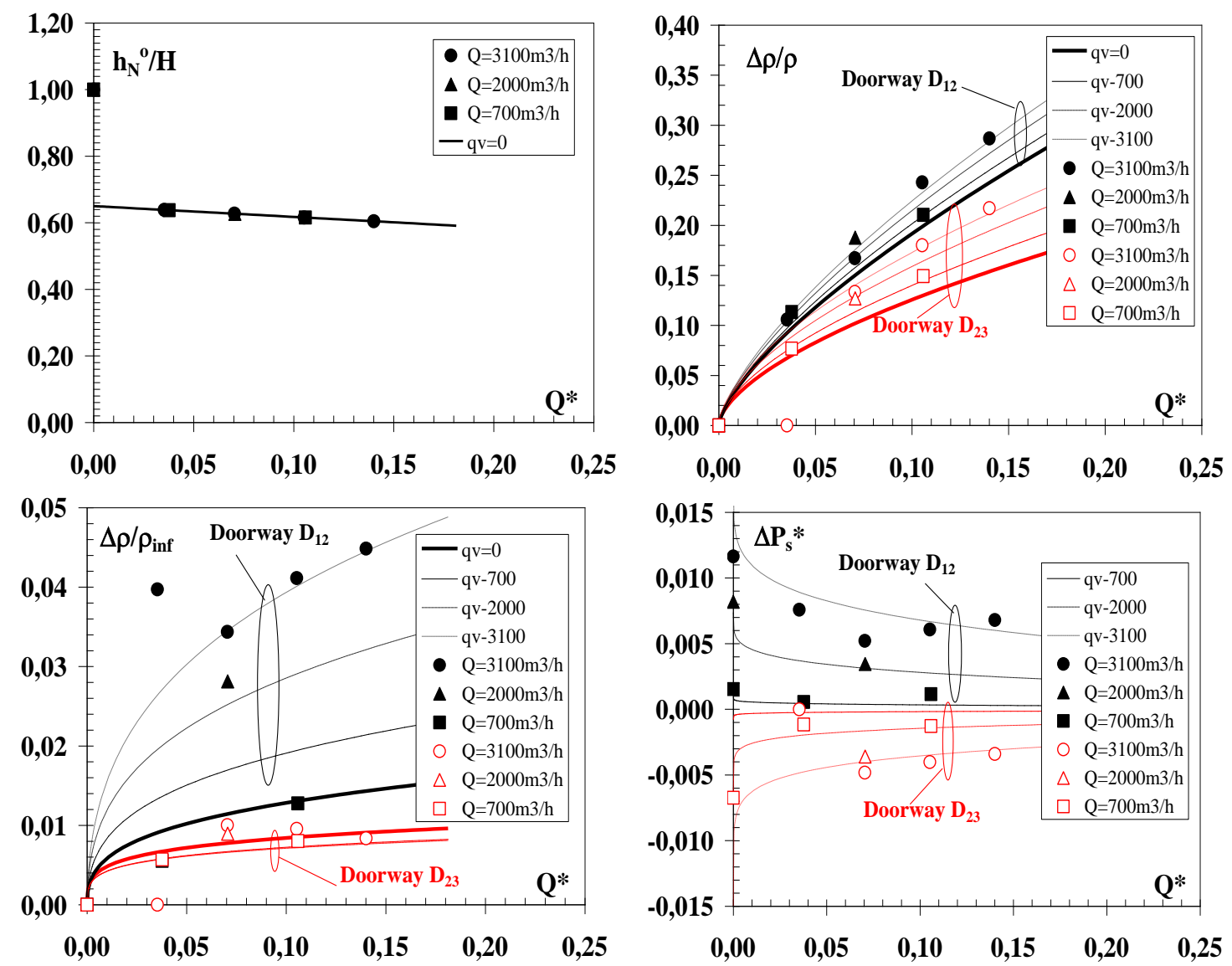

Fig. 11. Variation of parameters $\Delta \rho / \rho, \rho_{\text {inf }} / \rho, \mathrm{h}_{\mathrm{N}}{ }^{0} *$ and $\Delta \mathrm{P}_{\mathrm{s}} *$ versus fire HRR and ventilation rate.

\section{Top and Bottom Velocities}

The behavior of the maximum inflow and outflow velocities is presented in Fig. 12. The main effect of the fire HRR concerns the velocity of the outflow $\left(U_{H} *\right)$ (increase up to 0.6$)$. Its influence on the inflow $\left(U_{0} *\right)$ is of second order (increase of up to 0.25 only). The main effect of the forced ventilation flow rate concerns the flow that has the same direction as the ventilation flow; i.e., the inflow $\left(U_{0}{ }^{*}\right)$ for doorway $\mathrm{D}_{12}$ and the outflow $\left(U_{H} *\right.$ for doorway $\mathrm{D}_{32}$.

The proposed correlations match satisfactorily the experimental data. This result was expected since the coefficients of the correlations have been determined so that the correlations of the parameters $\Delta \rho / \rho, \rho_{\text {inf }} \rho$ and $\Delta P_{s}^{*}$ fit the data. Nevertheless, the good agreement means that the formulas given in Eq. 8 represent a good model of the physical phenomena. The advantage of these correlations is to predict behavior not tested experimentally such as the configuration without forced ventilation (large solid lines in the figures).

The correlations also show the existence of a critical ratio of ventilation flow rate and fire heat release rate for which the outflow velocity at the upstream doorway $D_{12}$ or the inflow velocity at the downstream doorway $\mathrm{D}_{23}$ are zero. This critical ratio can be used to determine the ventilation regime that avoids releasing smoke in the upstream room. For instance, with a ventilation rate of $3,100 \mathrm{~m}^{3} / \mathrm{h}$, a fire of HRR lower than 0.03 (dimensionless value) will not release smoke in the upstream room. 

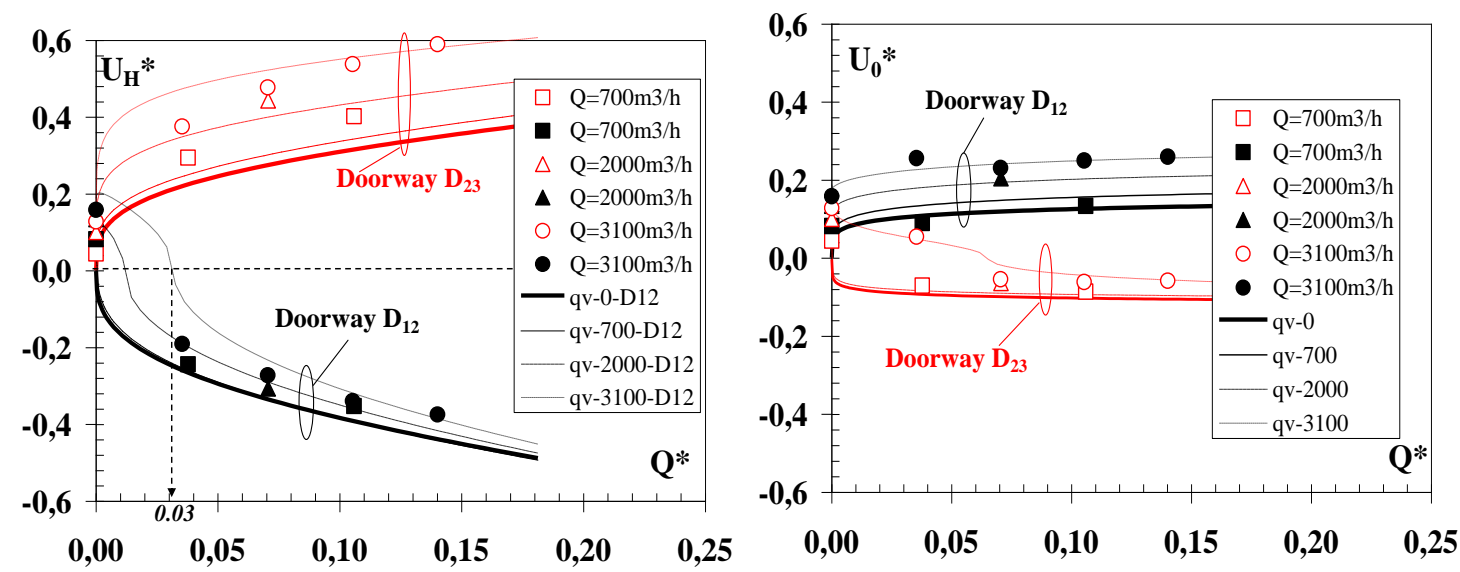

Fig. 12. Maximum velocities of the in and outflow for doorways as a function of fire HRR and ventilation rate.

\section{Neutral Plane}

The effects of the fire HRR and the ventilation flow rates on the location of the neutral plane are presented in Fig. 13. Firstly, the neutral plane in the upstream doorway $\left(D_{12}\right)$ is always higher than that in the downstream doorway $\left(D_{23}\right)$. As observed previously on the velocity profiles, the mechanical ventilation contributes to move up the neutral plane of the upstream doorway $\left(D_{12}\right)$ and to move down the one of the downstream doorway $\left(D_{23}\right)$. This effect is more important for the downstream doorway $D_{23}$.

As for the velocities, the first term in the expression of the neutral plane (Eq. 2) containing, $h_{N}{ }^{0}{ }^{\circ}$, represents the effect of the natural ventilation induced by the fire and is identical for the two doorways. It corresponds to the solid line in Fig. 13. Its value is about 0.65 and decreases slightly with the fire HRR. However, this level is large compared those calculated from formulations available in the literature [17] (between 0.3 and 0.5 ). The second term of the correlation represents the coupling effect of fire and ventilation and is different for the two doorways.

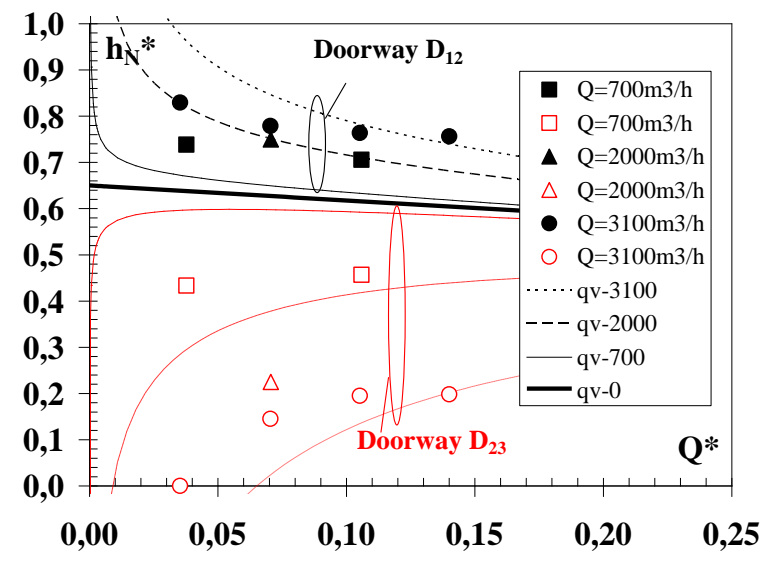

Fig. 13. Neutral planes for doorways function of fire HRR and ventilation rate.

\section{Mass Flow Rates}

The mass flow rates of the inflow and outflow are presented in Fig. 14. The outflow is larger for doorway $\mathrm{D}_{23}$ than for doorway $\mathrm{D}_{12}$. The ventilation increases the outflow toward the downstream room $L_{3}$ and limits the one toward the upstream room $L_{1}$. As expected, the opposite behavior is noticed for the inflow.

As for the mass flow rates, a correlative approach is proposed based on their relationship to the other variables. The following expressions are used: 


$$
\begin{array}{lll}
\dot{m}_{o}^{*}=k_{m}^{12} \frac{C_{d}}{2} U_{H}^{*}\left(1-\min \left(1 ; h_{N}^{*}\right)\right) \frac{\rho}{\rho_{\infty}} & \text { and } \quad \dot{m}_{i}^{*}=C_{d} U_{0}^{*} \min \left(1 ; h_{N}^{*}\right) & \text { for doorway } \mathrm{D}_{12} \\
\dot{m}_{o}^{*}=k_{m}^{23} \frac{C_{d}}{2} U_{H}^{*}\left(1-\max \left(0 ; h_{N}^{*}\right)\right) \frac{\rho}{\rho_{\infty}} & \text { and } \quad \dot{m}_{i}^{*}=C_{d} U_{0}^{*} \max \left(0 ; h_{N}^{*}\right) & \text { for doorway } \mathrm{D}_{23}
\end{array}
$$

The doorway coefficient, $C_{d}$, is taken as 0.6 for both doorways. For the outflow, a coefficient $\mathrm{k}_{\mathrm{m}}$ is needed to correct the simplicity of this relation and to satisfy the mass conservation. For the present study the value of the coefficient is 14 for both doorways. The value for this coefficient is large, a result which might be linked to the simplicity of the model that will have to be improved in future work. For the inflow, the simple relation matches satisfactorily with the experimental data. No correction is needed.

The mass flow rates are also compared to the usual literature relation [17] $0.5 A H^{0.5}$ (dotted line in Fig. 14) that corresponds to situations with flashover gas temperature in the fire room. As expected, the present experiments give much lower values.
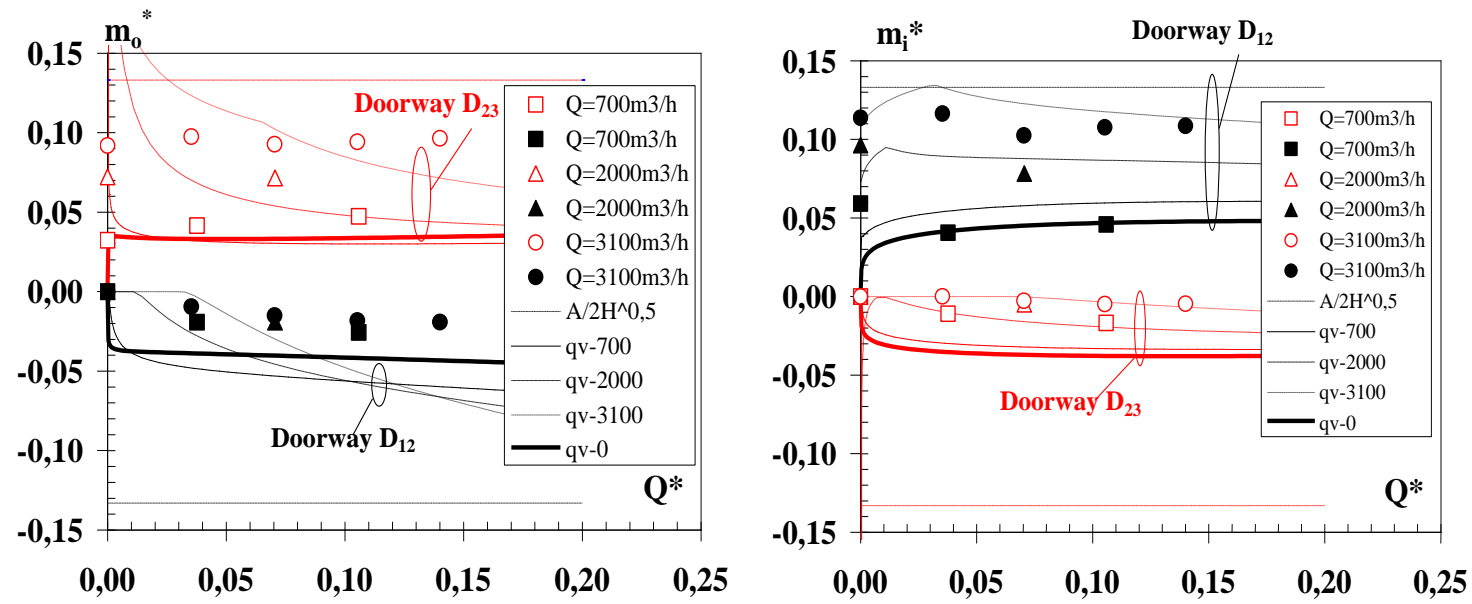

Fig. 14. In and out flow rates for doorways as a function of fire HRR and ventilation rate.

\section{CONCLUSIONS}

This study, which is based on large-scale experiments, points out several issues regarding smoke flow at doorways in the event of a fire scenario in confined and mechanically ventilated rooms.

The mechanical ventilation has a significant effect on doorway flows. It influences the inflow or outflow and also modifies the position of the neutral plane. The most important parameter is the ventilation flow rate. It contributes to a shift in the velocity profile and, therefore, modifies the in and out flows. A second important parameter is the direction of the ventilation flow with respect to the relative position of the doorway and the fire (doorway upstream or downstream). The flow in the doorway will display a different behavior depending on whether it is located upstream or downstream of the fire. When the fire is positioned upstream of the doorway, it behaves as a barrier reducing the effect of the ventilation.

A correlative approach is proposed for analyzing the results; it points out the coupled effect of ventilation and fire. The correlations give correct trends regarding the effect of ventilation and fire on the neutral plane and inflow and outflow velocities. These correlations allow for calculations of situations not tested experimentally. For instance, they can identify critical conditions for which the forced ventilation can stop the spread of smoke in the upstream room.

The present study presents experimental results analyzed with a correlative approach that can be used as a data base for the validation of code simulations. 


\section{REFERENCES}

[1] Mowrer, F.W., (2009), Driving Forces for Smoke Movement and Management, Fire Technology, 45: 147-162, http://dx.doi.org/10.1007/s10694-008-0077-1

[2] Lidwell, O.M., (1997), Air Exchange Through Doorways, The Effect of Temperature Difference, Turbulence and Ventilation Flow, Journal of Hygiene, 79: 141-154, http://dx.doi.org/10.1017/S0022172400052931

[3] Dalziel, S.B. and Lane-Serff, G.F., (1991), The Hydraulics of Doorway Exchange, Journal of Building and Environment, 26:121-135, http://dx.doi.org/10.1016/0360-1323(91)90019-8

[4] Quintiere, J.G. and DenBraven, K., "Some Theoretical Aspects of Fire Induced Flows Through Doorways in a Room-Corridor Scale Model”, NBSIR 78-1512, Gaithersburg, MD, 1978.

[5] Prahl, J. and Emmons, H.W., (1975), Fire Induced Flow Through an Opening, Combustion and Flame, 25: 369-385, http://dx.doi.org/10.1016/0010-2180(75)90109-1

[6] Zukosky, E.E., Kubota, T., and Lim, C.S., "Experimental Study of Environment and Heat Transfer in a Room Fire. Mixing in Doorway Flows and Entrainment in Fires Plumes", Report NBS-GCR85-493, Gaithersburg, MD, NIST, 1985.

[7] Nakaya, J., Tanaka, T., Yoshida, M., and Steckler, K., (1986), Doorway Flow Induced by a Propane Fire, Fire Safety Journal, 10: 185-195, http://dx.doi.org/10.1016/0379-7112(86)90015-9

[8] Phillips, J.C. and Woods, A.W., (2004), On Ventilation on Heated Room Through a Single Doorway, Journal of Building and Environment, 39:241-253, http://dx.doi.org/10.1016/j.buildenv.2003.09.002

[9] Wang, L. and Quintiere, J.G., (2009), An Analysis of Compartment Fire Doorway Flow, Fire Safety Journal, 44:718-731, http://dx.doi.org/10.1016/j.firesaf.2009.02.001

[10] Wang, L. and Quintiere, J.G., (2009), A General Formula for the Prediction of Vent Flows, Fire Safety Journal, 44:789-792, http://dx.doi.org/10.1016/j.firesaf.2009.03.012

[11] Stavrakakis, G.M. and Markatos, N.C., (2009), Simulation of Airflow in One and Two Enclosures Containing Fire Source, International Journal of Heat and Mass Transfer, 52: 2690-2703, http://dx.doi.org/10.1016/j.ijheatmasstransfer.2007.10.046

[12] Chow, W.K. and Zou, G.W., (2005), Correlation Equations on Fire-Induced Air Flow Rates Through Doorway Derived by Large Eddy Simulation, Building and Environment, 40:897-906, http://dx.doi.org/10.1016/j.buildenv.2004.09.010

[13] Bryant, R.A., (2009), A Comparison of Gas Velocity Measurements in a Full-Scale Enclosure Fire, Fire Safety Journal, 44: 793-800, http://dx.doi.org/10.1016/j.firesaf.2009.03.010

[14] Prétrel, H. and Audouin, L. "Smoke Movement Induced by Buoyancy and Total Pressure between two Confined and Mechanically Ventilated Compartments", Proceedings of the 2010 Interflam conference, Interscience Communications Limited, 2010, pp1053-1065.

[15] Beyler, C.L., 1991. Analysis of Compartment Fires with Overhead Forced Ventilation. Fire Safety Science 3: 291-300. http://dx.doi.org/10.3801/IAFSS.FSS.3-291

[16] Prétrel, H. and Such, J.M., (2005), Effect of Ventilation Procedures on the Behaviour of a Fire Compartment Scenario, Nuclear Engineering and Design, 235:2155-2169, http://dx.doi.org/10.1016/j.nucengdes.2005.03.003

[17] Karlsson, B. and Quintiere, J.G., Enclosure Fire Dynamics, CRC Press, (2000). 